\title{
Drugs abuse and increase in referral to hospital to prevent Recurrence of diabetic foot ulcer infection
}

\author{
Reasearch Article
}

\author{
Mostafa Madmoli ${ }^{1}$, Yaghoob Madmoli ${ }^{2}$, Fariba Mobarez ${ }^{3}$, \\ Hosein Taqvaeinasab ${ }^{4}$, Pouriya Darabiyan ${ }^{3}$, Alireza Rafi ${ }^{5^{*}}$
}

1. Emergency Medical Technician, Dezful University of Medical Sciences, Dezful, Iran.

2. MSc Student of Nursing, Student Research Committee, Ahvaz Jundishapur University of Medical Sciences, Ahvaz, Iran.

3. Student Research Committee, Ahvaz Jundishapur University of Medical Sciences, Ahvaz, Iran.

4. Student Research Committee, Dezful University of Medical Sciences, Dezful, Iran.

5. Student Research Committee, School of Nursing \& Midwifery,

Shahid Beheshti University of Medical Sciences, Tehran, Iran.

\begin{abstract}
Introduction: Diabetic foot ulcer is one of the complications of diabetes. This study was aimed to determine drugs abuse and increase in referral to hospital to prevent recurrence of diabetic foot ulcer infection. Materials and Methods: In this retrospective cross-sectional analytical descriptive study, 1693 patients with diabetes between 2015-17 were enrolled. Files of this number of diabetic patients admitted to khatam-ol-Anbia hospital in shoushtar city were studied. Data were entered into SPSS software version 18 and analyzed using descriptive statistics, analytical tests. Results: In this study 1693 patients with diabetes mellitus with a mean age of $52.13 \pm 53.22$ years. In the case of diabetic foot ulcers, $9.5 \%$ of the patients had diabetic foot ulcers and $4.8 \%$ had a history of amputation and $2.4 \%$ of the patients had a history of surgery on their diabetic ulcer. In this study, a significant relationship was found between education level and diabetic foot ulcer $(\mathrm{p}<0.003)$. Also, there was a significant relationship between limb amputation and drug abuse or smoking $(\mathrm{P}=0.009)$. In this study, patients who had drug and smoking or smoking $4.3 \%$ more than those who did not consume, they were referred to the hospital to prevent recurrence of foot ulcer infection. In this study, there was a significant relationship between drug abuse or smoking and the rate of surgery in diabetes mellitus $(\mathrm{P}=0.007)$. Conclusion: Given that in this study, patients who had drug and smoking or smoking $4.3 \%$ more than those who did not consume, they were referred to the hospital to prevent recurrence of foot ulcer infection. In this study, there was a significant relationship between drug abuse or smoking and the rate of surgery in diabetes mellitus. Therefore, there is a suggestion to reduce the consumption or abandonment of drugs and smoking.
\end{abstract}

Keywords: Diabetes, Drug abuse, Diabetic foot ulcer, Recurrence of ulcer infection, Smoking.

\section{Introduction}

Diabetes is one of the most commonly diagnosed diseases of metabolic disorders and is a global challenge $(1,2)$. It is a chronic, metabolic and genetically heterogeneous disease characterized by increased levels of blood glucose and carbohydrate metabolism disorders, protein and lipids. Inappropriate combination (low physical activity and unhealthy foods) has led to an uncontrollable increase in the prevalence of diabetes in the world. Complications of diabetes are very common among patients (3-7). In diabetic patients, depression is one of the most common psychiatric disorders (8). One of the most common and debilitating problems of youth and adolescents is

*Corresponding Author:

\section{Alireza Rafi,}

Student Research Committee,

School of Nursing \& Midwifery,

Shahid Beheshti University of Medical Sciences,

Tehran, Iran

E-mail: alirezarafi72@gmail.com depression and is so wide spread that among mental disorders it is referred to as a mental cold. $(9,10)$. Depression and daily occupational stress may cause some disorders in mental, and physical health of patients (11). The high occupational stress status is known as a psychosocial factor in cardiovascular disease (12). Diabetes mellitus also is one of the most common endocrine complications in thalassemic patients (13). Thalassemia syndrome is a hereditary blood disease. In general, the annual incidence of thalassemia disease is estimated at one in 100,000 worldwide (14-18). Due to late and dangerous complications of diabetes, it has been paying more attention every day. The disease has spread in the late 20th century and there is currently no sign of stopping it (19). The diabetes association refers to the disease as a growing, costly and cancer-related disease and a major health concern (20). Patients with diabetes are at greater risk than the general population of the growing urinary tract, liver, biliary, pancreatic, colon, endometrial, and kidney cancer. Several confounding factors are directly related to the clinical differences in diabetes at various levels of metabolic 
control, diabetes duration, anti-diabetic treatment profiles, and the presence of complications or association with disease (21). Diabetic foot ulcer is another complication of diabetes. Foot ulcer is a major complication of diabetes mellitus with high morbidity, mortality, and associated costs (22). The risk of having foot ulcer in diabetic patient is high (23). These foot ulcers are often infected with diabetic patients and have potential for cellulite development and if it is treated quickly and appropriately, it leads to blood and gangrene infections, and sometimes leads to amputation (24).

Because some previous studies have shown that diabetic patients' knowledge about the disease is very weak, in the study, Javadi et al., 58.3\% had poor knowledge (25). The lack of follow-up and prevention of diabetic foot ulcers will cause more infections and amputations. As well as drug abuse and smoking may increase the risk of diabetic ulcer infections, and that little research has been done on diabetic foot ulcers in Khuzestan province. Therefore, this study was conducted on diabetic patients admitted to Khatam-olAnbia hospital in Shoushtar, Khuzestan province, aimed to determine drugs abuse and increase in referral to hospital to prevent recurrence of diabetic foot ulcer infection.

\section{Materials and methods}

In this study, which evaluated 1693 diabetes patients and a retrospective cross-sectional descriptive study, records of this number of diabetic patients admitted to Khatam-ol-Anbia Hospital in Shoushtar city were studied. The patients who were diagnosed with diabetes and had a history of the disease that referred to Khatam-ol-Anbia hospital, Shoushtar, from 2015 to 2017, were entered in the study.

This article is the result of the research project of Behbahan University of Medical Sciences with the code IR.BHN.REC.1397.9533. After obtaining the necessary permissions and financial support from Behbahan University of Medical Sciences, this license was referred to the research department of Shoushtar School of Medicine, then the Research Committee of Shoushtar School of Medicine issued the necessary permission to Khatam -ol-Anbia Hospital and then the patients were informed by informed consent written and their records were used for this study.

The data needed for the study were extracted from patients' medical records during the years mentioned in the medical records section of the hospital. The inclusion criteria included all patients with a medical diagnosis and a history of any type of diabetes, and in each age group, and the exit criteria included other records of patients who had non-diabetic medical diagnosis as well as incomplete cases. In order to study the files and collect data, a written letter of introduction was submitted by the Vice-Chancellor for education and research of Shoushtar University of Medical Sciences.
Then, the files of patients referring to Khatam-ol-Anbia Hospital in the archives section were used. The required information was collected through a researcher checklist from the records.

The data in this study included demographic and clinical data of patients such as gender, age, BMI, BS, marital status, occupation, economic status, educational level, having or not having diabetic foot ulcer, limb amputation, history of surgery on diabetic ulcer, Family history of diabetes, having or not having a nephropathy, duration of diabetes, type of anti-diabetes drug, number of hospital referral for ulcer infection, history of drug abuse or smoking. Then data were entered into SPSS software version 18 and were analyzed using descriptive statistics including enumerated tables, mean, standard deviation and variance, and analytical tests including $\mathrm{T}$ test, ANOVA, chi-square and chisquare Pearson and at the significant level of $\mathrm{P}<0.05$.

\section{Results}

In this study 1693 individuals with diabetes mellitus with a mean age of $52.13 \pm 53.22$ years. Of these, $956(56.4 \%)$ were male and the rest were female. (Figure 1)

The mean BMI in these individuals was $25.13 \pm$ 2.03 , indicating patients with overweight. Also, the average blood glucose level in these patients was $221.43 \pm 83.71$.

In this study, 1005 (59.3\%) of the patients had A lower degree than a diploma and the rest had diplomas and higher. Also, 66.3\% were married, 12.4\% were widows, and the rest were single. In terms of economic situation, $40.4 \%$ of the people with poor financial status were, $22.3 \%$ average and the rest had a good economic situation.

Also in terms of diabetic foot ulcer, 9.5\% had diabetic foot ulcers and $4.8 \%$ had diabetes induced amputations and $2.4 \%$ of the patients had a history of surgery on their diabetic ulcers. (Figure 2)

In examining the proteinuria performed for patients (24-hour urine protein screening), 79.6\% normal and the rest were diabetic nephropathy. Of which $20.4 \%, 15.6 \%$ mild and $4.8 \%$ were severe. There was a significant relationship between diabetic nephropathy with age $(\mathrm{p}=0.008)$ and type of antidiabetic medicine $(\mathrm{p}=0.009)$ and diabetic foot ulcer $(\mathrm{p}=0.006)$.

Also, 213 people (12.5\%) had a family history of diabetes and the rest did not. Also, 209 (12.3\%) had a history of drug abuse or smoking. (Figure 3)

ANOVA test showed a significant relationship was found between education level and diabetic foot ulcer ( $p$ < 0.003). Also, there was a significant relationship between limb amputation and drug abuse or smoking $(\mathrm{P}=0.009)$, that means people who had more drugs or tobacco had more history of limb amputation.

In this study, patients who had drug abuse or 
smoking, $4.3 \%$ more than the non-consumer referred to the hospital to prevent recurrence of foot ulcer infection.

Chi-square test showed there was a significant relationship between drug abuse or smoking and the rate of surgery in diabetes mellitus $(P=0.007)$. In the same way, patients who were more to use drugs or tobacco, had more surgery on their ulcers.

In this study, there was no significant relationship between BMI with diabetic foot ulcer and limb amputation $(\mathrm{P}=0.05)$.

Figure 1: Frequency of males and females in diabetic patients.

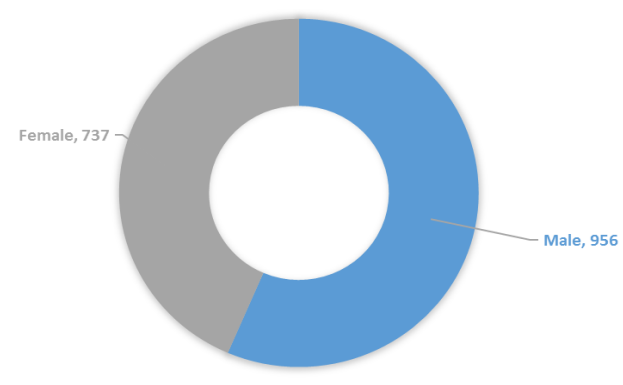

Figure 2: Frequency comparison of diabetic patients with a history of diabetic foot ulcer, limb amputation and surgery history on diabetic ulcer according to gender.

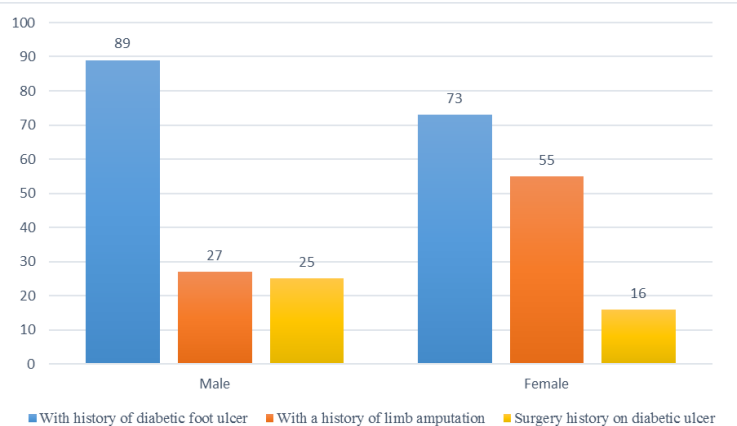

Figure 3: Frequency of family history of diabetes and history of drug abuse or smoking in patients with diabetes According to gender.

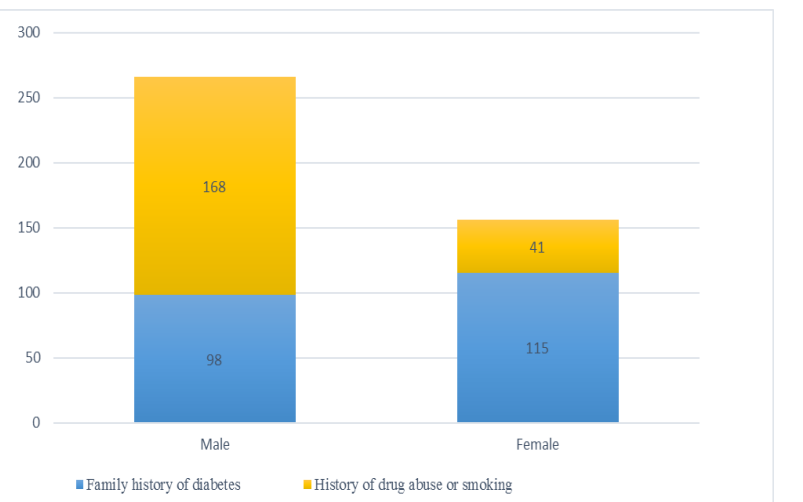

\section{Discussion}

The most common cause of hospitalization of diabetic patients is diabetic foot ulcer (26) So that about $50 \%$ of diabetics are admitted to the hospital due to diabetic foot problems (27). Because some previous studies have shown that diabetic patients' knowledge about the disease is very weak, (25) and that the lack of follow-up and prevention of diabetic foot ulcers will cause more infections and amputations. As well as drug abuse and smoking may increase the risk of diabetic ulcer infections, and that little research has been done on diabetic foot ulcers in Khuzestan province, therefore, this study was performed on diabetic patients admitted to Khatam-ol-Anbia Hospital in Shoushtar, Khuzestan province.

In this study, $9.5 \%$ of the patients had diabetic foot ulcers. In the study of Madmoli et al. (4), the prevalence of lower limb ulcer was $9.1 \%$. In another study, the prevalence of foot ulcer was $7.2 \%$, which is in line with the results of this study (28). In another study, it was $0.34 \%$ (29). Considering the different ulcer prevalence in the present study with some studies, it can be attributed to the effective prevention and treatment of foot ulcers, as well as the important role of genetics, lifestyle, nutrition and education.

Most people with diabetes in this study were male. In many studies $(4,30,31)$, most people with diabetes were women, that was not consistent with the present study. But in the study of Frykberg et al., 90.3\% of the population was male (32) and in another study, the number of men and women was equal (33). In many studies, the population of diabetic women is greater than men, which may be attributed to their gender characteristics, but in terms of complications of diabetes, men are more likely to become infected.

In this study, $12.5 \%$ of the patients had a family history of diabetes and the rest did not. Diabetes can greatly increase the chance of developing cancer. In a study entitled diabetes and the risk of cancer, in men, the risk of overall cancer has increased by $27 \%$ in those with a history of diabetes. HR was very high for people with liver, pancreatic and kidney cancer. This study demonstrated an increased risk of colon cancer and gastric cancer with borderline significance. In women, the incidence of cancer was significantly increased at the borderline, while the incidence of gastric cancer and liver cancer was statistically significant and the incidence of ovarian cancer was observed at the border (34). The association between diabetes and primary liver cancer in a case-control study in Italy in 428 confirmed cases of cancer cell carcinoma with histology, 59 cases with gall bladder cancer and 1502 cases was in the hospital for diseases non-acute, that sixty-four cases of hepatocellular carcinoma have been reported against 87 diabetes mellitus (35).

In this study, there was no significant relationship between BMI with foot ulcer and limb amputation. In many studies of diabetes, obesity and high BMI 
increased the risk of obesity-related cancer. The results of the study showed that adults overweight and glucose increased the risk of obesity-related cancer twice, while overweight adults with normal glucose increased the risk by $50 \%$. Adults with normal weight with increased glucose did not have the risk of excessive cancer (36).

In total alcohol consumption and drug abuse increases the risk of infection with diabetic foot ulcers and various diseases. In this study there was a significant relationship between limb amputation and drug abuse or smoking. That is, people who had more drugs or tobacco had more history of limb amputation. In this study, patients who had drug abuse or smoking, $4.3 \%$ more than the non-consumer referred to the hospital to prevent recurrence of foot ulcer infection. In this study, there was a significant relationship between drug abuse or smoking and the rate of surgery in diabetes mellitus, In the same way, patients who were more to abuse drugs or tobacco, had more surgery on their ulcers. The results of some studies demonstrated women with estrogen receptor-positive breast cancer, consumers received alcohol seven times or more per week compared to those who did not consume, $90 \%$ had a higher risk of developing maladaptive breast cancer (37). Another study also found that alcohol consumption was associated with lower survival (38). Recently, some cohort meta-analysis studies showed that there was a $17 \%$ increased risk of recurrence of cancer among the highest and lowest alcohol users (39). Other studies also found that increased mortality from breast cancer or recurrence with moderate to high alcohol drinking was observed $(40,41)$.

In a study, the prevalence of diabetic nephropathy among smokers (more than 10 cigarettes a day for more than a year) was higher by non-smokers compared to $19.2 \%$ versus $12.1 \%$. With increasing smoking, the frequency of nephropathy increases. No difference was found in the prevalence of proliferative retinopathy between smokers and non-smokers. Overall, the study concluded that smoking is a risk factor for developing nephropathy in people with type 1 diabetes (42). Also, in another study, there was no relationship between the risk or severity of retinopathy and the number of daily smoking cigarettes or the number of years of smoking compared to diabetes. These data suggest that there is no risk of retinopathy in smokers or with a history of smoking compared to those who have never smoked (43).

\section{Conclusion}

Diabetic foot is associated with an increased risk of death in diabetic patients. The most common cause is neuropathy, foot deformity, foot trauma, upper foot pressure and peripheral vascular disease that leads to lower limb amputation if not treated properly that is associated with an increase in mortality, high costs for treatment and a decrease in quality of life.
Given that in this study, patients who had drug abuse and smoking $4.3 \%$ more than those who did not consume they were referred to the hospital to prevent recurrence of foot ulcer infection. In this study, there was a significant relationship between drug abuse or smoking and the rate of surgery in diabetes mellitus. Therefore, there is a suggestion to reduce the consumption or abandonment of drugs and smoking. It is also better that the ministry of Health through the media, as well as the medical team, and especially the nurse, who have direct and close links with the patient, had a planning to help stop drug abuse and improve diabetic foot ulcer.

\section{Acknowledgment}

The authors of this article appreciate and thank all those who have co-operated with us.

\section{Conflict of interest}

There are no conflicts of interest in this article.

\section{References}

1. Moslemirad M, Madmoli M, Madmoli Y, Niksefat M. Prevalence of type 1 and type 2 diabetes and its related factors in diabetic patients hospitalized in Khatam-ol-Anbia hospital in Shoushtar, 2014-15: A retrospective study. Journal of Research in Medical and Dental Science. 2018;6(3):421-6.

2. Madmoli M, Eilami O, Rezaie K, Aliabad MA, Moslemirad M. Diabetes and the risk of suffering cardiovascular Diseases: A two-year retrospective study. International Journal of Ecosystems and Ecology Science (IJEES). 2018 Jun;8(3): 649-56.

3. Shirali M, Madmoli Y, Roohafza J, Karimi H, Baboli Bahmaei A, Ertebati S. Improvement Diagnosis of Diabetes Using a Combination of Sugeno Fuzzy Inference Systems and Firefly Algorithms. Iranian Journal of Diabetes and Metabolism. 2017; 15 (3) :172-176.

4. Madmoli M Rostami F, Mirsami Yazdi N, Mosavi A, Baraz Sh. Evaluation of Prevalence of Diabetic Foot Ulcer and Its Related Factors in Diabetic Patients Admitted to KHatam-ol-Anbia Hospital in Shoushtar During 2015-2016: A Retrospective Study. International Journal of Ecosystems and Ecology Science (IJEES). 2018 June; 8 (3): 545-52.

5. Rostami F, Madmoli M, Mirsami Yazdi N, Baraz Sh. Evaluation of The Prevalence of Lower Limb Amputation and Its Related Factors in Diabetic Patients Admitted to KHatam-ol-Anbia Hospital in Shoushtar During The 2015-2016: A Retrospective Study. International Journal of Ecosystems and Ecology Science (IJEES). 2018 June; 8 (3): 553-60.

6. Raisifar Z, Afshar Nia A, Maghamesi Moarrefi H, Madmoli M. Evaluation of gi Bleeding Prevalence and Its Related Factors in Diabetic Patients Hospitalized in KHatam-ol-Anbia Hospital During 
2015-16: A Retrospective Study. International Journal of Ecosystems and Ecology Science (IJEES). 2018 June; 8 (3): 609-14.

7. Mostafa Madmoli, Zahra Kord, Azita Bandani, Negin Sedighi, Mahla Rezaei Shandiz, Pouriya Darabiyan, Alieh AfsharNia. Epidemiological and clinical study of patients with Alzheimer's in Five Cities of Khuzestan Province in 2016-2018. Medical Science, 2019, 23(95), 1-5

8. Larijani B, Khoramshahee M, Bandarian F, Akhondzadeh S. Association of depression and diabetes in the doctor Shariati diabetes clinic and Iranian Diabetes Association. Iranian Journal of Diabetes and Metabolism. 2004 May 15;3(1):7782.

9. Madmoli Y, Madmoli M, Qashqaei nezhad N, Bosak S. Prevalence of depression and associated factors in adolescents of masjedsoleyman. JPEN. 2016; 2(4):31-40. [in Persian]

10. Madmoli M, Madmoli Y, Bigdeli Shamloo MB, Etebari A, Mahmoodi Kouhi A, Azami M. The Relationship Between Depression and Religiousness in Female High School Students of Masjed Soleyman in 2015. Journal of Pediatric Nursing. 2017 Jun 15;3(4):15-22.

11. Madmoli M, Nikpay S. An Investigation of the Relationship between Spiritual Health and Depression, Anxiety, and Stress among Students of Ilam University of Medical Sciences. Journal of Research in Medical and Dental Science. 2018 May 17;6(3):294-300.

12. Gheisari Z, Beiranvand R, Karimi A, Ghalavandi S, Soleymani A, Madmoli M, Bavarsad AH. Relationship between Occupational Stress and Cardiovascular Risk Factors Determination: A Case-control Study. Journal of Research in Medical and Dental Science. 2018 May 17;6(3):287-93.

13. Azami M, Sayehmiri K. Prevalence of diabetes mellitus in Iranian patients with thalassemia major: a systematic review and meta-analysis. Journal of Mazandaran University of Medical Sciences. 2016 Oct 15;26(141):192-204.

14. Madmoli Y, Beiranvand R, Korkini N, Mashalchi $\mathrm{H}$, Karimi H. [Comparison of health related quality of life in beta thalassemia major and healthy people in Dezful in 2015 (Persian)]. Iran J Nurs Res. 2016; 11(1):9-16.

15. Madmoli Y, Akhaghi Dezfuli SM, Beiranvand R, Saberi Pour B, Azami M, Madmoli M. [An epidemiological and clinical survey of patients with $\beta$-thalassemia in dezful in 2015 (Persian)]. Iran J Epidemiol. 2017; 13(2):145-52.

16. Maraghi E, Adavi A, Madmoli Y, HeidariSoureshjani R, Madmoli M. The Effect of Orem Self-Care on Mental Health of Patients with Thalassemia Major. Journal of Clinical Nursing and Midwifery. 2018;4(3).

17. Madmoli M, Madmoli Y, Rahmati P, Adavi A, Yousefi N, Gheisari Z, Abbaszade Aliabad M. Quality of Life and Some Related Factors in Patients with Beta Thalassemia Major in Southwest Iran. Journal of Client-centered Nursing Care (JCCNC. 2017;3(2).

18. Madmoli Y, Akhaghi Dezfuli SM, Adavi A, Maraaghi E, Heidari Soureshjani R, Madmoli M. The Effect of Orem Self-Care on Mental Health of Patients with Thalassemia Major. Journal of Clinical Nursing and Midwifery. 2018 Jul 1;7 (2):108-15.

19. Hamadzadeh S, Ezatti ZH, Abedsaeidi ZH, Nasiri N. Coping Styles and Self-Care Behaviors among Diabetic Patients. IJN. 2013; 25 (80) :24-33. \}in Persian\}.

20. Malek M, Aghili R, Emami Z, Khamseh ME. Risk of cancer in diabetes: the effect of metformin. ISRN endocrinology. 2013 Sep 24;2013.

21. E.Giovannucci,D.M.Harlan, M. C. Archer et al., "Diabetes and cancer: a consensus report," CA Cancer Journal for Clinicians, vol. 60, no. 4, pp. 207-221, 2010.

22. Bus SA, Armstrong DG, RW van Deursen, Lewis J EA, Caravaggi CF, Cavanagh PR. IWGDF guidance on footwear and offloading interventions to prevent and heal foot ulcers in patients with diabetes. Diabetes Metab Res Rev. 2016; 32(1): 2536. doi: 10.1002/dmrr.2697

23. Aghakhani N, Broomand A, Alinejad V, Torabi M, Nikoonejad A. The effect of education on quality of life in patients with diabetic foot in educational hospital of urmia. The Journal of Urmia Nursing and Midwifery Faculty. 2016;14(4):380-8.

24. Moeini M, Shahriari M, Yousefi H, Esfandiari J, Babaahmadi M. An investigation on the wound severity and its association with predisposing factors in patients with diabetic foot. Journal of Clinical Nursing and Midwifery. 2017;5(4).

25. Basri A, Motamed N, Mazinani N. Evaluation of Knowledge, Attitude and Practice of Diabetic Patients about Periodontal Disease in BushehrC in 2015-16. ISMJ. 2018 Jan 15;20(6):553-61.

26. Aallaa M, Sanjari M, Shahbazi S, Shayeganmehr Z, Abooeirad M, Amini MR et al. Diabetic foot workshop: Improving technical and educational skills for nurses. Med J Islam Repub Iran. 2017; 31.8. doi: 10.18869/mjiri.31.8.

27. Mukendi I, Mundawarara S, Luvhengo T. Factors to delayed presentation of patients with diabetic foot sepsis at Regional hospitals in the Ekurhuleni metropolitan municipality of Gauteng province. $\mathrm{S}$ Afr J Surg. 2017 Jun; 55(2):68.

28. Dekker RG, Qin C, Ho BS, Kadakia AR. The effect of cumulative glycemic burden on the incidence of 
diabetic foot disease. J Orthop Surg Res. 2016 Nov18; 11(1): 143. doi: 10.1186/s13018-016-047-y

29. Stoekenbroek RM, Lokin JLC, Nielen MM, Stroes ESG. How common are foot problems among individuals with diabetes? Diabetic foot ulcers in the Dutch population. Diabetologia. 2017; 60: 12711275. doi: $10.1007 / \mathrm{s} 00125-017-4274-7$

30. Urbančič Rovan V, Rovan J. An exploration of diabetic foot screening procedures data by a multiple correspondence analysis. Slovenian Journal of Public Health. 2017 Mar 1; 56(1):65-73. doi: 10.1515/sjph-2017-0009

31. Stoekenbroek RM, Lokin JLC, Nielen MM, Stroes ESG. How common are foot problems among individuals with diabetes? Diabetic foot ulcers in the Dutch population. Diabetologia. 2017; 60: 12711275. doi: 10.1007/s00125-017-4274-7

32. Frykberg RG, Gibbons GW, Walters JL, Wukich DK, Milstein FC. A prospective, multicentere, open -label, single-arm clinical trial for treatment of chronic complex diabetic foot wounds with exposed tendon and/or bone: positive clinical outcomes of viable cryopreserved human placental membrane. Int wound j. 2016; 14: 569-577. doi: 10.1111/ iwj. 12649

33. Tan JH, Hong CC, Shen L, Tay EY, Lee JK, Nather A. Costs of Patients Admitted for Diabetic Foot Problems. Ann Acad Med Singapore. 2015 Dec; 44(12): 567-70.

34. Inoue M, Iwasaki M, Otani T, Sasazuki S, Noda M, Tsugane S. Diabetes mellitus and the risk of cancer: results from a large-scale population-based cohort study in Japan. Archives of internal medicine. 2006 Sep 25;166(17):1871-7.

35. La Vecchia C, Negri E, Decarli A, Franceschi S. Diabetes mellitus and the risk of primary liver cancer. International journal of cancer. 1997 Oct 9;73(2):204-7.
36. Moore LL, Chadid S, Singer MR, Kreger BE, Denis GV. Metabolic health reduces risk of obesityrelated cancer in framingham study adults. Cancer Epidemiology and Prevention Biomarkers. $2014 \mathrm{Jul}$ 10: cebp-0240.

37. Li CI, Daling JR, Porter PL, et al: Relationship between potentially modifiable lifestyle factors and risk of second primary contralateral breast cancer among women diagnosed with estrogen receptorpositive invasive breast cancer. J Clin Oncol 27: 5312-5318, 2009

38. Walter V, Jansen L, Ulrich A, et al: Alcohol consumption and survival of colorectal cancer patients: Population-based study from Germany. Am J Clin Nutr 103:1497-1506, 2016

Schwedhelm C, Boeing H, Hoffmann G, et al: Effect of diet on mortality and cancer recurrence among cancer survivors: A systematic review and meta-analysis of cohort studies. Nutr Rev 74: 737748, 2016

40. Kwan ML, Kushi LH, Weltzien E, et al: Alcohol consumption and breast cancer recurrence and survival among women with early-stage breast cancer: The life after cancer epidemiology study. J Clin Oncol 28:4410-4416, 2010

41. Holm M, Olsen A, Christensen J, et al: Prediagnostic alcohol consumption and breast cancer recurrence and mortality: Results from a prospective cohortwith awide range of variation in alcohol intake. Int J Cancer 132:686-694, 2013

42. Telmer S, Christiansen JS, Andersen AR, Nerup J, Deckert T. Smoking habits and prevalence of clinical diabetic microangiopathy in insulindependent diabetics. Acta Medica Scandinavica. 1984 Jan 12;215(1):63-8.

43. Klein R, Klein BE, Davis MD. Is cigarette smoking associated with diabetic retinopathy? American journal of epidemiology. 1983 Aug 1;118(2):22838. 